\title{
"This Girl is on Fire"
}

\section{Sensemaking in an Online Health Community for Vulvodynia}

\author{
Alyson L. Young \\ Indiana University, IUPUI \\ Indianapolis, Indiana \\ youngaly@iupui.edu
}

\author{
Andrew D. Miller \\ Indiana University, IUPUI \\ Indianapolis, Indiana \\ andrewm@iupui.edu
}

\begin{abstract}
Online health communities (OHCs) allow people living with a shared diagnosis or medical condition to connect with peers for social support and advice. OHCs have been well studied in conditions like diabetes and cancer, but less is known about their role in enigmatic diseases with unknown or complex causal mechanisms. In this paper, we study one such condition: Vulvodynia, a chronic pain syndrome of the vulvar region. Through observations of and interviews with members of a vulvodynia Facebook group, we found that while the interaction types are broadly similar to those found in other OHCs, the women spent more time seeking basic information and building individualized management plans. They also encounter significant emotional and interpersonal challenges, which they discuss with each other. We use this study to extend the field's understanding of OHCs, and to propose implications for the design of self-tracking tools to support sensemaking in enigmatic conditions.
\end{abstract}

\section{CCS CONCEPTS}

- Human-centered computing $\rightarrow$ Empirical studies in collaborative and social computing.

\section{KEYWORDS}

Online health communities; sensemaking; enigmatic disease; vulvodynia; self-tracking

\section{ACM Reference Format:}

Alyson L. Young and Andrew D. Miller. 2019. "This Girl is on Fire": Sensemaking in an Online Health Community for Vulvodynia. In CHI Conference on Human Factors in Computing Systems Proceedings

Permission to make digital or hard copies of all or part of this work for personal or classroom use is granted without fee provided that copies are not made or distributed for profit or commercial advantage and that copies bear this notice and the full citation on the first page. Copyrights for components of this work owned by others than the author(s) must be honored. Abstracting with credit is permitted. To copy otherwise, or republish, to post on servers or to redistribute to lists, requires prior specific permission and/or a fee. Request permissions from permissions@acm.org. CHI 2019, May 4-9, 2019, Glasgow, Scotland UK

(C) 2019 Copyright held by the owner/author(s). Publication rights licensed to ACM.

ACM ISBN 978-1-4503-5970-2/19/05 .. \$15.00

https://doi.org/10.1145/3290605.3300359
(CHI 2019), May 4-9, 2019, Glasgow, Scotland UK. ACM, New York, NY, USA, 13 pages. https://doi.org/10.1145/3290605.3300359

\section{INTRODUCTION}

Online health communities (OHCs) allow people living with a shared diagnosis or medical condition to offer advice, seek support, and gather information directly from peers. In the last decade, OHCs have grown in popularity and scope. In addition to dedicated sites such as TuDiabetes [26] and PatientsLikeMe, OHCs have also formed as sub-forums or groups on larger social network sites, such as Reddit [14] and Facebook [17].

The HCI, Health Informatics, and CSCW communities have devoted substantial attention to OHCs, and in general they are well characterized across multiple studies, platforms, and diseases. OHCs allow people to seek out information about their condition, engage in sensemaking around the condition as it manifests in their daily lives, and provide various types of social support, such as informational and emotional support. However, less is known about the role of $\mathrm{OHCs}$ in enigmatic diseases, where the etiology is unknown or complex.

One such enigmatic condition is Vulvodynia, a chronic pain syndrome of the vulvar region. Women experiencing vulvodynia feel severe pain or discomfort in the vulva that lasts for at least three months [10]. Vulvodynia is a diagnosis of exclusion with no clear pathology, and its causes and treatments typically vary from woman to woman. Beyond physical pain, vulvodynia also causes severe psychological distress, impacting a woman's quality of life.

In this paper, we present findings from an observation and interview study of a vulvodynia Facebook group. We sought to understand how women with vulvodynia gather together online to collectively make sense of their condition.

This paper makes several contributions to HCI. We show that while the types of interaction in the group are broadly similar to those found on other OHCs, the enigmatic nature of vulvodynia means women spend much more time seeking basic information about their condition and building individualized treatment plans. Women in our study predominantly use the site to engage in knowledge-sharing, as a way to collectively define vulvodynia, figure out ways to improve their 
own situation, and devise strategies to be taken seriously by medical professionals. The participants also encounter significant emotional and interpersonal challenges in their daily lives, which they discuss and help each other through on these sites. We then discuss implications for sensemaking in enigmatic conditions, including ways technology might facilitate discussion and sensemaking through self-tracking.

\section{VULVODYNIA: AN ENIGMATIC PAIN CONDITION}

Vulvodynia is a chronic pain condition in women, characterized by severe pain or discomfort in the vulvar region that lasts for at least three months [10]. It is a prevalent condition, estimated to affect $16 \%$ of women at some point in their lives [1], and is often associated with comorbidities [3]. It affects women of all ages and ethnicities [16].

Despite its prevalence, vulvodynia is an enigmatic condition, lacking a clear etiology or definitive cause $[1,2]$. It is a diagnosis of exclusion with no clear pathology [2]. Women with vulvodynia experience a range of symptoms including soreness, burning, stinging, rawness, throbbing, and/or itching in the vulvar region [36]. While in some cases the vulvar tissue looks swollen or inflamed, it is more common for the vulva to appear normal.

The pain associated with vulvodynia can either be localized to a particular area of the vulva or generalized. The onset of vulvodynia can either be primary, with first genital contact, or secondary, after a period of pain free contact, and may be intermittent or persistent. Further, pain sensations may be provoked on touch, unprovoked, or mixed [7].

Although the causes of vulvodynia remain elusive, it is widely accepted that multiple etiologies are likely involved. Possible etiologies include injury to or irritation of the nerves surrounding the vulvar region, past vaginal infections (e.g., chronic thrush or bacterial vaginosis), allergies or sensitive skin (e.g., vulvar eczema or psoriasis), hormonal changes, or muscle spasms and weakness of the pelvic floor $[2,11]$.

In addition to physical pain, vulvodynia is also associated with emotional and behavioral issues including depression, anxiety, sleep disturbances, sexual dysfunction, altered body image, relationship problems, and decreased quality of life $[1,11]$. In particular, women with vulvodynia often report feelings of inadequacy as a woman and sexual partner [5], often reinforced by dominant societal norms of femininity and societal pressures for coital relationships [37]. This leads to psychological distress, which can exacerbate physical pain.

Treatment options for vulvodynia vary, ranging from topical or oral medications to cognitive-behavioral therapy to pelvic floor physical therapy to injections or surgery $[1,12]$. However, due to limited randomized controlled trials, there is currently insufficient evidence to support the benefits of any specific intervention [12]. Thus, vulvodynia treatment remains a trial-and-error process, where different interventions are tried in an effort to reduce or eliminate pain.

\section{RELATED WORK}

In this section, we review the prior literature in three areas: online health communities, complex health concerns, and sensemaking in health informatics.

\section{Online Health Communities}

Online Health Communities (OHCs) are digital spaces in which people gather to discuss a given health concern [31]. They are usually comprised primarily of patients or people experiencing a medical concern and may be standalone sites sub-forums within a larger site or service, such as Reddit [14] or Facebook [17]. OHCs have been widely studied in HCI, CSCW, and Health Informatics. While they share much in common with other online communities, OHCs are distinct in their characteristics and goals. Research has consistently shown that these sites provide a space for individuals with common diseases and health concerns to seek out information about their condition and receive support from others like them [17, 30, 31]. Peer interaction in OHCs provides members with informational, emotional, and instrumental support for health and disease management [30, 35]. HCI researchers have also investigated ways to aid information seeking in OHCs through the integration of clinical expertise, via semi-automated techniques [20] and leveraging peer moderators to weed out misinformation and manage the community [19]

However, most of the research on OHCs focuses on diseases where the etiology and treatment are well-known, such as diabetes [18, 36] and cancer [33]. Less is known about the experiences and practices of OHCs when the mechanisms of the condition are poorly understood or recognized, as in complex health concerns.

\section{Complex Health Concerns}

We define complex health concerns as diseases or health conditions that are epidemiologically rare or under recognized and poorly understood in the medical literature. These conditions often lack a clear etiology, making them difficult to diagnose and treat effectively. Within HCI, CSCW, and Health Informatics, a body of work has begun to examine complex health concerns, such as fibromyalgia [9], endometriosis [29], irritable bowel syndrome [21], multiple sclerosis [32], rare diseases [24], and conditions that are often subject to misdiagnosis and treatment, such as prolonged symptoms of Lyme disease [28]. As part of this work, researchers have investigated online information seeking and behavior patterns [9], the extent to which support needs are met through $\mathrm{OHC}$ interactions [24], how competing viewpoints online affects individuals' understanding of their health condition [28], 
and how carers make decisions about which online health resources to use and trust [32].

HCI researchers have also examined the design and use of personal informatics tools for enigmatic and other complex diseases. Karkar et al., [21] for instance designed and evaluated a diagnostic self-tracking tool for patients with irritable bowel syndrome (IBS) to identify their food triggers. IBS is an enigmatic disease because the pathophysiology is unknown [8]. Researchers have also examined how to design self-tracking tools for learning at scale and for disease types, such as endometriosis, where it is unclear what data types are relevant to the disease [29]. Finally, researchers have examined the self-tracking practices of individuals with complex neurological diseases that cause physical, cognitive, and psychological symptoms [6]. We add to this body of work by examining another complex health concern, vulvodynia, and the ways that women with this enigmatic condition use $\mathrm{OHCs}$ to make sense of their condition.

\section{Sensemaking in Health Informatics}

Sensemaking is the process by which individuals come to understand complex social and environmental situations [34]. Within health informatics, the theoretical perspective of sensemaking has been advanced by Mamykina and colleagues [27] to better understand self-monitoring for chronic conditions such as diabetes. In their 'sensemaking perspective' framework, they focus on the ways that individuals "make sense of their disease, learn from past experiences, interpret new information and developmental models to inform their future choices" (p. 407). In this view, sensemaking consists of more than just one-time decisions or problem-solving to overcome specific barriers to self-management goals, but rather is focused on how individuals organize complex lived experiences to find patterns, discover connections and dependencies, and make daily choices for disease self-management. From this, three essential activities emerge: perception of new health and wellness information; inference to inform actions, and action based on new information. While this framework was developed within the context of diabetes, the authors suggest it may apply more broadly.

With different colleagues, Mamykina has also explored collective sensemaking within a social computing lens, in a study of online diabetes community TuDiabetes [26]. Through interviews with administrators and active users and a thematic analysis of discussion thread posts, the authors found that members of TuDiabetes construct shared meaning through deep discussions, negotiation of perspectives, and the resolution of conflicts of opinion. They also found that members valued multiplicity of opinions over consensus.

In this paper, we build on and extend this prior research on sensemaking in OHCs by examining how women with vulvodynia, an enigmatic pain condition, use social media to collectively make sense of their condition. Unlike diabetes, which has a very specific set of symptoms that are generally well understood in the medical literature and has defined standards of care in medical practice, vulvodynia has no clear etiology and there is insufficient evidence to support the benefits of any treatment. This study therefore examines the ways that women with vulvodynia co-construct an understanding of their condition, build personalized management plans for their individual symptoms, and devise ways to be taken seriously by medical professionals. Moreover, it examines how the uncertainty of the condition impacts participants' emotional and interpersonal well-being.

\section{METHODS \& APPROACH}

For this study, we conducted participant observations of one vulvodynia Facebook group and open-ended interviews with group members. The first author, who has vulvodynia, has been an active member of the vulvodynia support group since February 2018 and has been officially observing the group conversations since May 2018. Participant observations were conducted for 3 months, between May 17 and August 17, 2018. The first author engaged in conversations with the women, sharing her own experience and providing recommendations on what has worked for her. Detailed notes were taken on the general topics discussed with select examples captured in screen shots. This resulted in 125 pages of notes and approximately 40 hours of direct observation.

The participant observations were supplemented with 11 open-ended interviews. Participants were asked to share their journey with vulvodynia, sources of support, and use of technology in managing their condition. These interviews allowed us to further understand participants' experience with vulvodynia, their treatment strategies, and the role of social computing technology in understanding their condition. Interview participants were recruited from the vulvodynia Facebook group. They ranged in age from 23 to 65 years old, with a median age of 36.4. The majority of participants lived in the United States, with the exception of three from Australia, Canada, and Columbia, respectively. All women interviewed were heterosexual. Interviews lasted between 30 to 90 minutes and were conducted either online or by phone. All interviews were audio-recorded and transcribed. Participants received a 20.00 USD visa card for their participation, which they could opt to donate to a charity of choice.

The observation data were inductively coded to generate initial themes around participants' use of social media to understand, define, and discuss their condition. Further iterative analyses focused on the varied and individual practices that participants use to treat their condition, the challenges they face in interacting with providers and finding appropriate management plans, and the psychological impacts of the condition. The interview data were cross-referenced and 
Table 1: Interview Participant Demographics

\begin{tabular}{cccc}
\hline Participant ID & Age & Location & Relationship Status \\
\hline P1 & 23 & Australia & Boyfriend \\
P2 & 37 & United States & Married \\
P3 & NA & United States & Boyfriend \\
P4 & 25 & United States & Boyfriend \\
P5 & 30 & United States & Married \\
P6 & 23 & United States & Single \\
P7 & 65 & Canada & Married \\
P8 & 46 & United States & Married \\
P9 & 41 & United States & Married \\
P10 & 24 & Columbia & Single \\
P11 & 50 & United States & Married \\
\hline
\end{tabular}

deductively coded for the themes generated in the observations. The data for this paper are drawn primarily from the participant observations as supported by the interviews.

Ethics approval was obtained from Indiana University's Human Research \& Institutional Review Board prior to conducting the research. The first author contacted administrators for two private Facebook groups for permission to observe the group discussions. We were granted access to one of the two groups. A pinned message was posted to the group explaining the purpose of the study with an option to opt out. Members were informed that notes would be taken on general themes with select examples captured in screenshots, which would be immediately anonymized. Following concern from some group members, the first author also agreed to only capture screenshots from those members who had explicitly consented to the research, either by liking the pinned post or expressing their consent in the message thread. In a few cases, the first author contacted a group member privately to obtain permission to use a specific post.

\section{Research Site: Vulvodynia Support Group}

The vulvodynia support group observed for this study is a private Facebook group, which had approximately 1,300 members at the time of observation. We chose this particular group for our analysis because of the frequency of posts and replies, broad topics of discussion, and access. Members posted between 4-10 messages per day, with each post receiving at least 1 response or like, and more popular content receiving upwards of 40 replies.

\section{FINDINGS}

We found that women predominantly use the group to engage in knowledge-sharing to collectively define vulvodynia, construct individualized management plans, and devise strategies to be taken seriously by providers. They also encounter significant emotional and interpersonal challenges in their daily lives, which they discuss and help each other through on the site. We describe each of these in detail next, integrating findings from the interviews with participant observations to strengthen our points.

\section{Co-Constructing an Understanding of Vulvodynia}

Vulvodynia's obscure etiology meant that many women in the group struggled to determine the cause of their condition or if they had the condition in the first place. They therefore used the group to collectively make sense of their condition and to build a shared understanding of it. Women in the group would periodically ask others, sometimes as a poll, if they knew the cause of their condition: "Questions for all of you! When did you first discover you had pain? Was it one day to the next or was it gradual? For me it was one day to the next that things got awful."

In response to this type of query, the women who responded were often unsure of the exact cause; however, many had their assumptions. For example, some women believed that they developed the condition as a result of chronic infections, namely yeast, bacterial vaginosis (BV), and urinary tract infections (UTIs), and the overuse of antifungal creams and antibiotics to treat these infections. As one woman shared: "Mine came on suddenly I'd been taking antibiotics \& using a cream for a vaginal discharge and then the first flare started." In particular, ciprofloxacin (aka cipro), which is used to treat UTIs, was repeatedly referenced by the women as a leading potential cause of the condition. As one woman stated: "Why are they still prescribing Cipro? They are aware it causes damage yet still it's given out like candy. $S M H . "$ Others believed it was the result of hypertonic pelvic floor muscles or pudendal neuralgia, causing nerve pain in the vulvar region. One woman speculated that that her vulvodynia was caused by a 4 -wheeler accident, stating that her "pelvis was shattered which caused lots of nerve damage". Still others suspected a connection to traumatic birth.

In some cases, women were unsure if they had the condition before joining the group. They often learned of vulvodynia only after searching online for information on their pain symptoms, when swab tests came back negative for infections and doctors were unable to provide a diagnosis or answers. In these instances, they used the group to learn about the condition and to try to self-diagnose before seeing another specialist. New members often posted their symptoms to see if diagnosed others reported similarities. One woman shared, for instance, that since undergoing a medical procedure she had been experiencing a dry, raw, and irritated feeling that also presented as pins and needles and sent shooting pains when touched. While her doctor assumed an infection, all tests came back negative. Another woman with 
diagnosed vulvodynia shared that while she has not had the same procedure, she has similar symptoms, which she "often mistakes ...for infections. And the tests come back negative". She continues that her physical therapist mentioned, "pelvic floor tightness and dysfunction often occur in these cases due to trauma", which can cause nerve damage or muscle tightness, leading to vulvodynia symptoms.

As vulvodynia is often associated with comorbidities [3] such as fibromyalgia, interstitial cystitis, polycystic ovary syndrome, and irritable bowel syndrome - conditions that can also cause pelvic pain, the women sometimes had difficulty determining if their symptoms were related to vulvodynia or another condition. Examples in the observations included questions about whether there was a connection between vulvodynia and persistent genital arousal disorder, urinary frequency and bladder pain, or pelvic floor dysfunction. In one case, a woman asked about the differences between interstitial cystitis (IC: a painful bladder condition that causes urinary urgency and bladder and pelvic pain) and vulvodynia as she was trying to determine if she had developed the latter. Another woman shared that each syndrome causes pain symptoms in different parts of the body:

IC is burning on my bladder and urethra, leaving me with UTI symptoms but not always having a UTI ... Vulvodynia is any pain within the vulva or vagina, it can be the tissues or in the muscles. I get an acid burning when my vestibulitis flares.

Rather than rely solely on textual descriptions, another approach was to annotate the location of pain on a medical diagram. This provided an added visual aid, helping to identify women with similar symptoms who could suggest potential causes or possible treatment options. In Figure 1, for instance, a woman highlighted an area where she experiences a rash or razor-burn feeling and she questions if it could be nerve pain. Other women offer her possible causes and suggestions, such as it "could be overactive nerves" which is "definitely worth looking into" or that a "pelvic floor health eval will tell if there is some pelvic floor dysfunction going on."

The women also sought advice on possible triggers; things that might increase their pain or bring on a flare. One woman, for example, asked whether there was a connection between pain and emotional state, to which other woman confirmed that anxiety and depression can make pain symptoms worse. While in another instance, a woman asked about the link between pain and foods as she was experiencing increased pain since eating something spicy. Another women responded that she is unable to eat anything spicy because her "bladder and vagina just start burning all at once. So awful. It usually lasts a few days". The women then used this information to avoid (as in the case of food) or reduce (as in the case of stress) these potential triggers in the future.

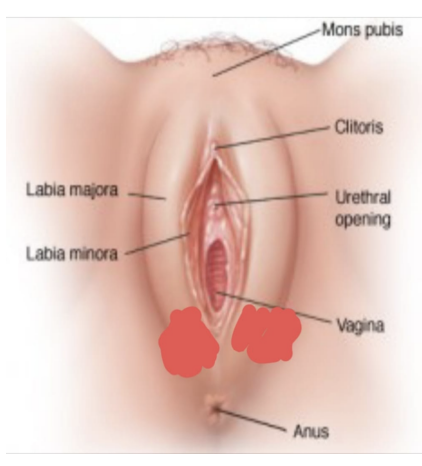

Figure 1: Annotated image indicating location of pain (used with permission).

\section{Building an Individualized Management Plan}

In the process of collectively making sense of their condition, women in the group worked to build an individualized management plan for their specific symptoms and suspected underlying causes. In the interviews, nearly all participants reported using the Facebook group primarily to find information on treatments and possible cures. For example, P1 mentioned the group enabled her to learn about non-medical treatments:

I never would have thought to change my laundry detergent, change my fabrics of the clothes I wear. I was never told that stuff. The physicians and gynecologists haven't said it. I think because [women on the forums] are dealing with these issues on a day to day basis ...they know the everyday little things that aren't medical that can help you manage this effectively.

Unlike other chronic diseases where treatment options are well-established and researched (e.g., diabetes), vulvodynia is treated through a process of trial-and-error. This means that most women have to try different treatment options before finding one or several that work to alleviate their symptoms. This reality of vulvodynia led many of the women in the group to comment, 'the journey to healing is very individual'. That is, each woman has a very different experience with each remedy they try, and what works for one person may not work for others. They would often preface this when offering advice or sharing their experiences with a particular treatment. Despite this, the women used the group predominantly to identify possible treatments and to ask others about their successes with a particular modality, intervention, or medication, or to receive advice on how to deal with a particular symptom. As one interviewee mentioned: "I like hearing the personal experiences of how medications and other products work for people because there's no other resource to hear how these are working" (P6). Thus, the most common use of 
the group was to ask about specific medications or medical interventions, either that they had been prescribed or that they were considering asking their doctors about.

For example, one woman in the group shared that her "GP has just given me Estriol cream via an applicator to try and help me with vulvodynia" and wondered if others had been prescribed this treatment and if it had worked. Other women shared their personal experiences, with some stating that they had used it and it helped, particularly for those who are menopausal, while others indicated it caused increased burning. Unless a woman had a particularly horrific reaction to a specific treatment, most would recommend trying it to see if it worked. For example, in a different conversation, one woman encouraged a group member "not to give up before trying it", stating that if she has a "negative reaction you can stop using it." This reflects the individualized nature of vulvodynia and its treatment. Even in those instances of a reported an adverse effect, women often shared their experiences with the caveat that each person is different and others may respond more positively than they did.

In addition to asking questions about a medication they had been prescribed, women would also request information about particular treatments that they had heard about to elicit feedback from others. A common question was 'have you tried X, and if so, did you find relief?' During the observations, one of the most frequently asked questions was about the use of CBD oil for pain relief: "is cbd any good?" The prevalence of this particular question was likely due to recent changes in the legalization of CBD oil in the US, opening another door for the women to try to find some relief from their pain.

Women also used the group to ask for assistance during a flare, often sharing what they had already tried and seeking other possible suggestions for addressing their symptoms. One woman, for instance, reported severe swelling of the vestibule and was looking for ideas for quick relief, stating that she had tried "the usual coconut oil, sitz/epsom baths, antihistamines, and ice packs but was wondering what everyone else does for relief when the swelling gets really bad". Other women offered their recommendations for longer term solutions, such as taking omega 3 supplements or using a steroid cream to help reduce the inflammation. By contrast, one woman suggested a more immediate solution that involved using an analgesic ointment on a tampon to reach the "internal area where the vestibule cause[s] the most pain." While she notes that getting the tampon in is uncomfortable ... the numbing payoff is worth it". Thus, the women used the group to help each other work through their pain by providing solutions that have worked well for them in the past.

Self-management plans extended beyond medications. Additionally, the women often sought advice on how to cope with everyday life. Normal activities, such as wearing tightfitted clothing, using feminine hygiene products, travel, or prolonged sitting typically resulted in added discomfort or brought on a flare. They sought workarounds that would enable them to live a fuller life, which included socializing with friends and family, maintaining a career, and enjoying leisurely activities. One woman, for example, asked for alternatives to riding a bike:

Hey, does anyone here use or knows of an alternative to the bike? I take the bus right now, but it's a real waste of money and time when the weather is nice. Unfortunately I can't bike because I have vulvodynia and pudendal neuralgia, but maybe someone here knows of equally healthy and eco-friendly options

Similar advice seeking was sought for items such as comfortable underwear, loose pants appropriate for work, workout clothing, and hygiene products that were sensitive enough to use near the vulva. Like above, the women in the group would share specific products that worked for them, often providing links to where to find the products online.

In both the observations and interviews, the women frequently mentioned that the physical and emotional toll of vulvodynia caused many women to seek out any and all treatments that might relieve their pain. For example, one interviewee stated: "I read a study that women in the U.S. spend 8,000 USD every 6 months on treatments for this condition. We will take anything because we in so much pain" $(\mathrm{P} 1$; emphasis ours). Similar comments were found in the observations. For instance, one woman, in response to a concern raised by another member about her sibling's approach to her condition, responded, "you just reach a point where you will do anything to make the pain go away". She then recommended that they look into pelvic floor physical therapy as this has helped many women.

\section{Self-Advocacy in a Patriarchal Healthcare System}

A core challenge for women with vulvodynia is locating a specialist with knowledge of the condition and a willingness to help [12]. Consequently, women in the observations often sought recommendations for specialists in their area (e.g., can anyone recommend a specialist in Dayton Ohio?) or feedback on specialists they were considering seeing, for example: "Got an appt with Dr. [redacted] in AZ!! For those of you that have seen him, what was your experience like?"

Women in the group frequently reported frustration with doctors who dismissed their concerns, failed to listen to them, or treated their pain as a psychological condition (i.e., "it's all in your head") rather than a physical one. There was a common belief among the women, in both the observations and interviews, that if this were a male problem, medical 
science would have figured it out by now (P1, P2). The women would frequently report that doctors 'don't listen' to their concerns and 'only prescribe medications to cover-up their symptoms' rather than looking for the root cause.

In line with this, the women reported feeling dismissed by doctors regarding the extent or reality of their pain. They often told stories about how practitioners would "throw their hands up and walk out of appointments saying they don't know how to help". One woman shared that her doctor did not believe her pain and effectively accused her of lying:

I have had at least one female doctor do that. She as much as accused me of lying about the condition because she had never heard of it. And every time I tried to say something about it, she cut me off and wouldn't let me speak.

Female doctors, in particular, were viewed by the women in the group as less empathetic than male physicians, and more likely to attribute their pain to a normal part of being a woman. One woman in the group shared that a female doctor once suggested that her cramps couldn't be that bad. When this happened, the women would encourage each other to seek out other healthcare providers who specialize in pelvic pain and would take their concerns more seriously. They would often recommend specific providers or types of clinics that have helped them in the past. While an extreme case, one woman reported 'ditching' all providers and 'taking her health into her own hands'.

This led many of the women to suggest they needed to be their own doctors and health advocates. In both the observations and interviews, women reported compiling their own research and bringing their findings with them to medical appointments to push for specific treatments. One woman in the group stated that her "own research became her rescue" and that "you have to educate yourself about your health issues and then demand the right therapy". This assertion was further echoed in the interviews by $\mathrm{P} 2$ who stated: "... we have to become essentially our own doctors because doctors are not helping. Letting us all down. They can't find a cause or cure. They have no clue; they just give us pills to go away".

This perceived need to take ownership of one's own health and health literacy was driven largely by the belief that providers, including gynecologists, have limited to no understanding of vulvodynia. Thus, there was a perceived risk of being prescribed the wrong treatment, such as antibiotics for an infection that wasn't there, or being given a prescription for a medication that hasn't worked in the past, as one woman in the observations experienced when a new doctor wrote her another script for an anti-depressant commonly used to treat nerve pain.

As part of this, women also frequently asked for advice on what questions to ask or what tests to request before seeing

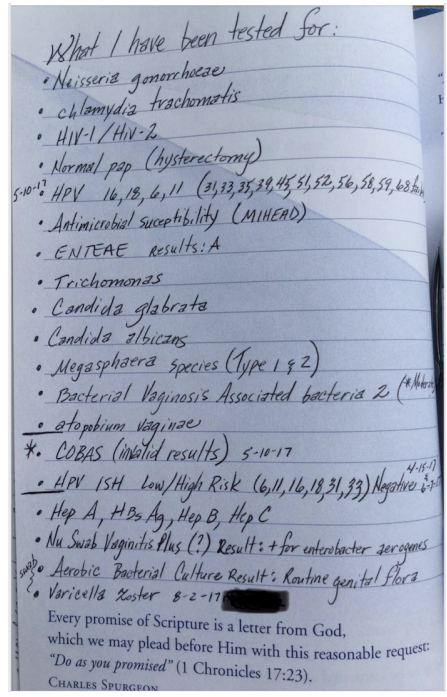

Figure 2: List of current tests (used with permission).

a new specialist. In some instances, they would also share what they had already had done. For example, one woman in the support group shared that she was about to go to her first doctor's appointment in nearly a year and provided an image of the tests she's already had done (Figure 2). She then asked others if there was anything else she should ask to be tested for. While some women provided recommendations for other tests, others commended her for taking time to document everything, as this would improve her appointment outcome:

I'm just here to say that I LOVE that you have a list. This is a really good idea it will make sure you don't forget things and to make sure you get all the information you're concerned about.

Through this process of knowledge-sharing, the women were able to bring comprehensive lists with questions and requested tests to their appointments and, in some cases, successfully push for additional diagnostics or treatments.

In line with this, the women in the interviews also used the group to learn about new treatment options and, sometimes, to push their providers for specific treatments. One interviewee, for instance, credited her progress with vulvodynia to using the information she'd collected from the group to demand specific procedures, despite her provider arguing that her symptoms did not fit the diagnostic criteria:

All my treatment options are just through all of these [Facebook] contacts. It's not been a physician telling me, 'hey, I think we should do a pudendal nerve block.' It's been me saying I have been your guinea pig for 30 flipping years and now it's time that I get to pick what I want to be a guinea pig for (P9). 


\section{Psychological Distress, Self-Preservation, \& Emotional Support}

Beyond physical pain, vulvodynia also causes extreme psychological distress, often leading to anxiety and depression, dyspareunia, poor self-image, and relationship problems $[1,11]$. While such discussions occurred much less frequently than posts related to informational support and knowledge sharing, when they happened they were significant. One woman in the observations shared, it feels like "someone stole my life from me". There was also a feeling that people who do not have the condition were unable to understand the physical and emotional turmoil that it causes: "[It] takes an emotional toll in a way that other people don't understand" (P5). In both the observations and interviews, the women reported that family and friends often did not believe or understand the extent of their pain. For instance, one interviewee stated:

People would look at you \& me and think 'there are these perfectly healthy young ladies' and you feel like a woman ... especially as a young woman it's hard to be taken seriously when you look like you're fine but it feels like your crotch is on fire ... you can't visibly see that. (P3)

The women therefore used the group to 'vent' and seek emotional support from others like them, often qualifying their posts with something to the effect of 'just looking for a friendly ear and someone who understands'.

Women in the group would occasionally post messages expressing their emotional state and how their pain made them feel: "My vag is on FIRE tonight, this sucks so bad" and "I just can't live like this anymore. I'm going crazy." Often, these messages were embedded in longer discussions about a particular symptom or treatment, rather than as an original post. For example, when discussing chronic infections, one woman mentioned that her pain gets so intense at times she feels as if she is 'losing her mind'. Not knowing if they will ever be pain free again leaves many women emotionally distressed and manic, particularly at the early stages of the condition. During the observations, a few women struggled to cope with the extent of their pain and the strain it was having on their psychological wellbeing and personal relationships, commenting to the effect the condition had 'destroyed their lives' and that they 'can't go on'.

During these times of distress, the women supported each other by offering emotional support, encouraging each other to "not give up" and to "hang in there". One interviewee mentioned: "just feeling that I'm not alone has been so important" (P1). Women in the group often offered their condolences and sympathy by stating, for example: "I am so sorry. I have had those pains. They are awful and knock you to the floor and I wouldn't wish them on anyone. Please know you are supported here." It was common for the women to let others know that they were "not alone" and that "healing is possible".

At its worst, the women sometimes commented that they are considering suicide. In the interviews, for example, P8 shared that she knew of at least two women who had taken their lives because of vulvodynia. When disclosed to the group, the women would encourage the individual to seek out psychological counseling, often providing the name or number for a local organization.

While the women would go out of their way to support someone in need, they had their limits. This was particularly the case if they felt the woman hadn't done enough to advocate for herself or to seek out appropriate treatment. In these cases, they might couch their support in 'tough love':

My heart goes out to you. I've seen you posting in complete desperation, but still is not clear to me that you have gone to a proper vulvar pain specialist to see what could be the CAUSE of your pain. You can't just simply shoot in the dark. People will give you all kinds of advice about what helps them, but it may not do anything for you because YOUR case needs to be evaluated ... Until you KNOW WHAT IS CONTRIBUTING, you will just be in the dark, please don't do this to yourself (emphasis in original).

In more extreme cases, women chose to block indviduals so that they would no longer their posts. This was done for self-preservation and self-protection. Topics such as suicidal ideation, comments made invalidating someone else's pain, or a deluge of posts caused some women to experience added stress. As one woman stated:"I felt badly that she was so desperate, as I know we have all been there or are there. But I had to block her, because I was getting such anxiety from seeing all her posts."

As in prior research [5], vulvodynia took a toll on how the women saw themselves, both as a woman and a partner. The women often struggled with feelings of inadequacy since intercourse was either challenging or impossible. One woman, for example, shared that she spent an entire day preparing herself for intimacy with her partner, only to be brushed off:

I planned a date night ... and spent time getting all fixed up and basically all day doing self care so that my pain would be low enough for sex tonight.. I've tried to initiate something multiple times and there's still something else he would rather be doing ... what do I do? I feel so worthless and like a terrible woman and girlfriend.

Some women in the group also blamed the failure of their relationships on vulvodynia, suggesting that their partners ended the relationship because they were unable to handle the condition and how it made them feel. Such comments 
were often met with sympathy, but also words of encouragement that they deserve someone who would stick by them through thick and thin: "I am so sorry. The same thing happened to me, and then next thing you know I met my fiancé and now we are getting married ...He's so understanding and kind. I promise that you deserve better."

Similar responses were given when women mentioned going out of their way to please their partner, despite their own comfort. One woman, for instance, asked for advice on cute, comfortable clothing after her husband requested that she dress nicer. While the women offered suggestions, they also encouraged her to continue wearing what makes her most comfortable and doesn't aggravate her pain: "Honestly, I would wear what makes you comfortable ... Your comfort should be the top priority... wanting you to look nicer around the house at the cost of your comfort possibly seems selfish"

\section{Ecosystems of Support}

The Facebook group examined in this study was just one of an ecosystem of social media platforms and other online resources the women used to understand and manage their condition. While this larger group provided a starting point for women to find others like them, it often failed to suffice more local or culturally-specific needs. The women therefore often joined or created new groups to support these needs. One interviewee, for example, reported using a local vulvodynia group for product recommendations that were available in her country (P1). Another shared she created a Facebook group focused on healing through diet (P2). During the observations, two new groups were also created to support the unique needs of Christians and LGBTQ, respectively. The women also used social media beyond Facebook. One interviewee, for example, shared she used Instagram for its uplifting messages (P4), while another shared she created a blog to document her experiences (P6). Beyond social media, the women also used resources from the National Vulvodynia Association (P4, P5), WebMD (P10), and Google searches (P6). We intend to examine this social media ecosystem in more detail in future research.

\section{DISCUSSION}

Our objective with this study was to understand how women with vulvodynia use OHCs to collectively make sense of their condition. We found the women predominantly used Facebook for knowledge-sharing; as a way to co-construct an understanding of their condition, build personalized management plans, and devise ways to be taken seriously by providers. We now compare how these sensemaking activities relate to the sensemaking framework for chronic illnesses proposed by [27]. We then discuss how sensemaking for enigmatic conditions could be facilitated through selftracking technology.

\section{Sensemaking in an Enigmatic Condition}

In [27]'s model, disease self-management is carried out in two modes, across three activities. In the sensemaking mode, individuals explicitly and effortfully engage in an analytical process of examining properties and constructing explanations of their situation to enable the selection of appropriate actions, while in the habitual mode, individuals utilize preexisting models that do not create new gaps in understanding. During these two modes, individuals monitor and classify new information and experiences related to their health and wellbeing (perception), develop and activate relevant internal representations for selecting appropriate courses of action (inference), and carry out daily activities in response to new information (action).

We chose this model because patients' experiences in vulvodynia share many similarities with diabetes. As in [27], we found that women with vulvodynia are thrown into an unfamiliar world with limited understanding of the condition and how it will impact their daily lives. Like diabetics, women with vulvodynia are faced with re-examining routine everyday activities and are forced to adjust their lives to effectively manage their condition. For our participants, this meant actively thinking about, for instance, the clothing they can wear, the feminine hygiene products they can use, how to negotiate intimate relationships, and how their emotional state might impact their condition. In the process, they experienced gaps in their understanding, which impacted their ability to select suitable actions and forced them to take steps to understand their new situation. Our study suggests one way the women did this was by using an OHC to collectively make sense of their condition as a way to define and explain their new reality.

While many similarities exist in sensemaking between diabetes and vulvodynia, including lifestyle adjustments and concerns around disease origin, there are core differences that suggest the model could be refined for enigmatic conditions. These include:

- Treatment Goals: in diabetes, patients' work towards maintaining their glycemic control, whereas in vulvodynia, patients' work to achieve highly individualized goals, such as an acceptable pain level, pain free sex, and maintaining employment; and,

- Outcome Goals: in diabetes, glucose levels (i.e., HbA1c) are an objective measure that needs to be in a certain range (approximately 6.5-8.5\%)[4]. By contrast, in vulvodynia, pain is highly subjective and what one woman considers reasonable may be disabling for another.

Further, in [27] the authors focus on a limited number of intervention modalities, namely diet, exercise, and medication and one outcome goal (i.e., glucose control); whereas 
in vulvodynia there are many more interventions, including medication, physical therapy, clothing, posture, laundry detergent, and ice/heat packs, and the outcome objective is highly individualized, such as achieving an acceptable pain level, intercourse, exercise, and wearing pants.

Sensemaking Mode in Vulvodynia. Our study suggests that these differences have implications for the sensemaking activities that occur around self-management for vulvodynia. For the women in our study, the fact that vulvodynia lacks an objective stabilizing factor that can be easily measured and monitored to provide feedback on the impact of an intervention (e.g., blood glucose monitor), meant that there was no systematic way for them to track what does and does not work. This was compounded by the fact that vulvodynia lacks a clear etiology and insufficient evidence exists to support the benefits of any particular intervention. Thus, women with vulvodynia must engage in sensemaking not only about their individual symptoms but about the condition itself. This affects both the individual sensemaking process and places additional urgency on women's ability to collectively make sense of their condition and derive and disseminate effective self-management strategies.

Consequently, the women in our study used the group to co-construct an understanding of vulvodynia. This gap in understanding often led them to share and ask others about their symptoms and potential underlying cause. While this might enable them to develop an internal representation of a suspected etiology (e.g., chronic infections), it did not always mean they were able to identify an appropriate or effective course of action. This was because vulvodynia is a highly individualized and even subjective experience. As our participants emphasized, each individual responds differently to different treatments, and what works for one individual may not work for others. This led them to use the information from the group to make educated guesses to drive their next course of action for managing their condition. These guesses were often based on suggestions from individuals with similar symptoms or potential underlying causes. In cases where a selected treatment was not effective, the women would return to the group to seek out other potential options, repeating the process.

When faced with new or increased pain, the women in our study would try to construct explanations for why they were experiencing these changes and what alternations they should make to reduce or eliminate their pain [27]. In our study, we observed women reflecting on their activities prior to the onset of symptoms and questioning, by posting to the group, if these activities could have caused their pain to flare. Recall the woman who asked about the connection between her emotional state and increased pain. Once a woman has lived with vulvodynia for a while, she starts to identify her pain triggers and uses this information to direct future actions in the self-management of her condition.

Although women may be able to identify triggers and modify their behavior to decrease their pain level, in most cases their overall pain symptoms continue to persist. Consequently, the sensemaking mode does not end when they complete this action. Rather, they continue to operate in this mode, sometimes for several years, as they try to construct explanations for their condition as a whole that will suggest which interventions will resolve their symptoms or stabilize their pain level. As we show, the women actively used the group to find and share ways to manage their condition.

Habitual Mode in Vulvodynia. The habitual mode refers to the default state where individuals maintain or attempt to return after engaging in sensemaking [27]. Many of the women in our study never fully reached this stage. For the few who did, this mode corresponded to when they found a treatment that worked and they had reached a manageable pain level or they considered themselves cured. In this stage, they often stopped experimenting with new interventions and fell into routine action. In our study, some of the women who reached this stage used their experiences to help others manage their condition, such as the woman who created a Facebook group to coach women on treating vulvodynia through diet (P2).

\section{Facilitating Sensemaking for Enigmatic Conditions through Self-Tracking}

In this section, we describe how vulvodynia patients could derive benefits from self-tracking technologies, echoing findings from other chronic conditions. We then expand on prior research to propose specific design considerations for vulvodynia support technologies. We also highlight opportunities for interpersonal health informatics tools to enable individual and collective sensemaking in enigmatic conditions.

While the women in our study often engaged in very deep discussions about their condition, the Facebook platform itself was not particularly well-suited for collective sensemaking. Similar to what [26] found in their study on collective sensemaking in an online diabetes forum, Facebook lacks affordances that enable the community, particularly new or occasional users, to effectively find discussions as they become buried below new content. Consequently, only those individuals who participated in the original discussion are privy to the conversations. In our study, this meant the same questions were asked repeatedly. Sometimes, women responded by suggesting an individual search for previous topics, rather than start a new discussion.

We suggest that self-tracking tools may provide a means to facilitate sensemaking for enigmatic conditions at both the individual and collective level. Self-tracking tools enable people to gain awareness of their behaviors in an effort to 
develop healthy lifestyle practices [13, 23]. For individuals with chronic conditions, such as chronic pain or rare diseases, such tools provide opportunities for self-discovery to drive targeted behavior changes to aid in self-management $[15,25]$, as well as increase individuals' sense of agency and control over their condition, often leading to a greater sense of wellbeing and quality of life [6]. When applied to the aggregate level, self-tracking tools also provide opportunities for information discovery across individuals, aiding in collective sensemaking [29].

The need for self-tracking tools also came from the women themselves. In both the interviews and observations, women bemoaned the lack of tools to adequately track, understand, and manage the unpredictable and multidimensional nature of vulvodynia. Consequently, they often relied on pen-andpaper based diaries (Figure 2, P6) to track this information.

Research has already begun to examine the ways that selftracking tools could be designed to support enigmatic and other complex health conditions where the causal mechanisms are poorly understood or challenging [6, 21, 22, 29]. In many ways, the prior findings align well with the needs of women with vulvodynia. For instance, previous research has demonstrated the importance of personalization in selftracking for enigmatic diseases [22, 29], arguing that it reduces the burden of use and provides more meaningful data about one's own condition and situation.

Personalization in self-tracking is particularly important for vulvodynia, a condition with high variability in patient experience, treatment, triggers, and diagnosis. To make sense of and manage one's unique experience with vulvodynia, self-tracking tools therefore need to support individual information needs and goals. For women with vulvodynia, this includes the ability to track the type, duration, location and intensity of pain; emotional, dietary, and lifestyle triggers; and, treatment efficacy.

The complex relationship between stimulus and patient experience in enigmatic conditions requires comprehensive tracking of potential triggers and treatments in order to be able to identify casual relationships which cannot easily be intuited [22]. For our participants, this meant trying to understand which triggers or treatments had either a positive or negative effect on their pain level. While research has mentioned the importance of trigger tracking for enigmatic conditions [21, 29], the impact of treatments on the patient experience has been largely ignored. In our study, participants frequently listed multiple simultaneous treatments and speculated openly about which was providing an improvement (i.e., decreased pain). Since treatment for vulvodynia is mostly empirical trial-and-error [12], the benefits of any particular intervention may not be acutely obvious to the patient. Further, some treatments have significant undesirable side effects impacting patients' willingness to continue treatment. Vulvodynia patients thus need to be able to not only track triggers that impact their experience but also the effect of treatments on their condition.

Our study also shows the potential for patient-generated data aggregation and synthesis to support information seeking and discovery. In our study, we identified three main information needs: shared understanding of symptoms, potential triggers, and treatment efficacy. While the ability to track these at the individual level is important for selfmanagement, often women with vulvodynia are unsure which triggers or treatments to consider. Self-tracking tools that provide information and filtered visualizations at the aggregate level may increase possibilities for collective sensemaking as well as the identification of other potential triggers and treatments not considered by the individual. Specifically, the ability to find women with similar symptoms and suspected underlying causes could aid individuals in identifying targeted self-management strategies. With sufficient data engagement, this could also potentially lead to naturalistic phenotypes for the condition [29] that extend beyond descriptions of provocation, location, and persistency of pain.

There is a unique need in vulvodynia for individual and collective sensemaking that enables patients to accurately describe the location and sensation of their symptoms at a very fine-grained scale. Currently, pain diaries only allow individuals to track pain symptoms to a general physical location and with few options to characterize the nature of pain. To accurately indicate the location of pain, women with vulvodynia need a high resolution and physically realistic image of the vulva, which they can annotate with the different pain sensations and intensities that are characteristic of the condition, such as burning, throbbing, stabbing, and itching. In a collective sensemaking context, this could also provide an aggregate level understanding of common symptom characteristics across the condition, leading to greater chances of diagnosis and/or eventual resolution.

Finally, self-tracking also offers opportunities for data sharing with providers as a way to educate them about the condition and help patients gain acceptance by the medical community. In future research, we intend to engage with providers to understand how such patient reported data could be used for enigmatic disease diagnosis and treatment. The inclusion of crowdsouced provider information could also aid individuals with vulvodynia and other enigmatic conditions in locating specialists who treat such conditions, something our research suggests is particularly challenging.

\section{CONCLUSION}

We contribute an understanding of how women with vulvodynia, an enigmatic pain condition, use $\mathrm{OHCs}$ to collectively make sense of their condition. We compare how the sensemaking activities for this enigmatic condition relate 
to sensemaking in other chronic illnesses where the mechanisms of the disease are well understood, illustrating similarities and key differences in how disease complexity impacts our understanding of sensemaking processes in disease selfmanagement. We then describe how self-tracking tools could be designed to better support sensemaking in enigmatic conditions, for both individual disease self-management and collective understanding of disease characteristics. We suggest that self-tracking at the collective level could aid in both initial diagnosis and drive eventual resolution in vulvodynia.

\section{ACKNOWLEDGMENTS}

We thank our participants for their contribution to this study. We also thank the reviewers for their feedback, Jessica Pater and Christina Chung for their comments on earlier drafts, and Neha Patil, Rachana Solanki, and Whitney Walker for their research support.

\section{REFERENCES}

[1] Meryl Alappattu, Georgine Lamvu, Jessica Feranec, Kathryn Witzeman, Michael Robinson, and Andrea Rapkin. 2017. Vulvodynia is not created equally: empirical classification of women with vulvodyndia. Journal of Pain Research 10 (Jul 2017), 1601-1609. https://doi.org/10.2147/JPR S136751

[2] Jose De Andres, Nerea Sanchis-Lopez, Juan Marcos Asensio-Samper, Gustavo Fabregat-Cid, Vicente L. Villanueva-Perez, Vicente Monsalve Dolz, and Ana Minguez. 2016. Vulvodynia-An Evidence-Based Literature Review and Proposed Treatment Algorithm. Pain Practice: The Official fournal of the World Institute of Pain 16, 2 (Feb 2016), 204-236. https://doi.org/10.1111/papr.12274

[3] Lauren D. Arnold, Gloria A. Bachmann, Sarah Kelly, Raymond Rosen, and George G. Rhoads. 2006. Vulvodynia: Characteristics and Associations with Co-Morbidities and Quality of Life. Obstetrics \& Gynecology 107, 3 (Mar 2006), 617-624. https://doi.org/10.1097/01.AOG. 0000199951.26822 .27

[4] American Diabetes Association. 2018. 6. Glycemic Targets: Standards of Medical Care in Diabetes-2018. 41, Supplement 1 (2018), S55-S64. https://doi.org/10.2337/dc18-S006

[5] Kathryn Ayling and Jane M. Ussher. 2008. If sex hurts, am I still a woman?: the subjective experience of vulvodynia in hetero-sexual women. Archives of Sexual Behavior 37, 2 (Apr 2008), 294-304. https: //doi.org/10.1007/s10508-007-9204-1

[6] Amid Ayobi, Paul Marshall, Anna L. Cox, and Yunan Chen. 2017. Quantifying the Body and Caring for the Mind: Self-Tracking in Multiple Sclerosis. In Proceedings of the 2017 CHI Conference on Human Factors in Computing Systems (CHI '17). ACM Press, New York, NY, 6889-6901. https://doi.org/10.1145/3025453.3025869

[7] Jacob Bornstein, Andrew T. Goldstein, Colleen K. Stockdale, Sophie Bergeron, Caroline Pukall, Denniz Zolnoun, Deborah Coady, consensus vulvar pain terminology committee of the International Society for the Study of Vulvovaginal Disease (ISSVD), the International Society for the Study of Women's Sexual Health (ISSWSH), and the International Pelvic Pain Society (IPPS). 2016. ISSVD, ISSWSH and IPPS Consensus Terminology and Classification of Persistent Vulvar Pain and Vulvodynia. Obstetrics \& Gynecology 127, 4 (Apr 2016), 745-751. https://doi.org/10.1097/AOG.0000000000001359

[8] Michael Camilleri. 2004. Treating irritable bowel syndrome: overview, perspective and future therapies. British fournal of Pharmacology 141,
8 (Apr 2004), 1237-1248. https://doi.org/10.1038/sj.bjp.0705741

[9] Annie T. Chen. 2012. Information seeking over the course of illness: the experience of people with fibromyalgia. Musculoskeletal Care 10, 4 (Dec 2012), 212-220. https://doi.org/10.1038/sj.bjp.0705741

[10] Claudia Chisari and Joseph Chilcot. 2017. The experience of pain severity and pain interference in vulvodynia patients: The role of cognitive-behavioural factors, psychological distress and fatigue. fournal of Psychosomatic Research 93 (2017), 83-89. https://doi.org/10. 1016/j.jpsychores.2016.12.010

[11] Mayo Clinic. 2018. Vulvodynia-Symptoms and causes-Mayo Clinic. https://www.mayoclinic.org/diseases-conditions/vulvodynia/ symptoms-causes/syc-20353423

[12] Kim J. Cox and Cynthia E. Neville. 2012. Assessment and management options for women with vulvodynia. Journal of midwifery and women's health 57, 3 (Jun 2012), 231-240. https://doi.org/10.1111/j.1542-2011. 2012.00162.x

[13] Daniel A. Epstein, An Ping, James Fogarty, and Sean A. Munson. 2015. A Lived Informatics Model of Personal Informatics. In Proceedings of the 2015 ACM International foint Conference on Pervasive and Ubiquitous Computing (UbiComp '15). ACM Press, New York, NY, 731-742. https: //doi.org/10.1145/2750858.2804250

[14] Jordan Eschler, Zakariya Dehlawi, and Wanda Pratt. 2015. SelfCharacterized Illness Phase and Information Needs of Participants in an Online Cancer Forum. In Proceedings of the Ninth International AAAI Conference on Web and Social Media. 101-109.

[15] Sergio Felipe, Aneesha Singh, Caroline Bradley, Amanda CdeC Williams, and Nadia Bianchi-Berthouze. 2015. Roles for Personal Informatics in Chronic Pain. In Proceedings of the 2015 ACM International Joint Conference on Pervasive and Ubiquitous Computing (UbiComp '15). ACM Press, New York, NY, 161-168. http://dl.acm.org/citation.cfm? id=2826165.2826189

[16] Bernard L. Harlow and Elizabeth Gunther Stewart. 2003. A populationbased assessment of chronic unexplained vulvar pain: have we underestimated the prevalence of vulvodynia? Journal of the American Medical Women's Association (1972) 58, 2 (2003), 82-88. https: //doi.org/10.1145/3068755.3068758

[17] Katrina L. Hinson. 2017. Framing Illness Through Facebook Enabled Online Support Groups. Communication Design Quarterly Review 4, 2b (Mar 2017), 22-31. https://doi.org/10.1145/3068755.3068758

[18] Jina Huh and Mark S. Ackerman. 2012. Collaborative Help in Chronic Disease Management: Supporting Individualized Problems. In Proceedings of the ACM 2012 conference on Computer Supported Cooperative Work (CSCW '12). ACM Press, New York, NY, 853-862. https: //doi.org/10.1145/2145204.2145331

[19] Jina Huh, David W. McDonald, Andrea Hartzler, and Wanda Pratt. 2013. Patient Moderator Interaction in Online Health Communities. AMIA Annual Symposium Proceedings (Nov 2013), 627-636. https: //www.ncbi.nlm.nih.gov/pubmed/24551364

[20] Jina Huh, David W. McDonald, Andrea Hartzler, and Wanda Pratt. 2014. Weaving Clinical Expertise in Online Health Communities.. In Proceedings of the SIGCHI Conference on Human Factors in Computing Systems (CHI '14). ACM Press, New York, NY, 1355-1364. https: //doi.org/10.1145/2556288.2557293

[21] Ravi Karkar, Jessica Schroeder, Daniel A. Epstein, Laura R. Pina, Jeffrey Scofield, James Fogarty, Julie A. Kientz, Sean A. Munson, Roger Vilardaga, and Jasmine Zia. 2017. TummyTrials: A Feasibility Study of Using Self-Experimentation to Detect Individualized Food Triggers.. In Proceedings of the SIGCHI Conference on Human Factors in Computing Systems (CHI '17). ACM Press, New York, NY, 6850-6863. https://doi.org/10.1145/3025453.3025480

[22] Ravi Karkar, Jasmine Zia, Roger Vilardaga, Sonali R. Mishra, James Fogarty, Sean A. Munson, and Julie A. Kientz. 2016. A framework for 
self-experimentation in personalized health. fournal of the American Medical Informatics Association 23, 3 (May 2016), 440-448. https: //doi.org/10.1093/jamia/ocv150

[23] Ian Li, Anind Dey, and Jodi Forlizzi. 2010. A Stage-based Model of Personal Informatics Systems. In Proceedings of the SIGCHI Conference on Human Factors in Computing Systems (CHI '10). ACM Press, New York, NY, 557-566. https://doi.org/10.1145/1753326.1753409

[24] Haley MacLeod, Grace Bastin, Leslie S. Liu, Katie Siek, and Kay Connelly. 2017. Be Grateful You Don't Have a Real Disease: Understanding Rare Disease Relationships.. In Proceedings of the 2017 CHI Conference on Human Factors in Computing Systems (CHI '17). ACM Press, New York, NY, 1660-1673. https://doi.org/10.1145/3025453.3025796

[25] Haley MacLeod, Anthony Tang, and Sheelagh Carpendale. 2013. Personal Informatics in Chronic Illness Management.. In Proceedings of Graphics Interface 2013 (GI '13). ACM Press, New York, NY, 149-156. http://dl.acm.org/citation.cfm?id=2532129.2532155

[26] Lena Mamykina, Drashko Nakikj, and Noemie Elhadad. 2015. Collective Sensemaking in Online Health Forums.. In Proceedings of the 2017 CHI Conference on Human Factors in Computing Systems (CHI '15). ACM Press, New York, NY, 3217-3226. https://doi.org/10.1145/ 2702123.2702566

[27] Lena Mamykina, Arlene M. Smaldone, and Suzanne R. Bakken. 2015. Adopting the sensemaking perspective for chronic disease selfmanagement. Journal of Biomedical Informatics 56 (Aug 2015), 406-417. https://doi.org/10.1016/j.jbi.2015.06.006

[28] Jennifer Mankoff, Kateryna Kuksenok, Sara Kiesler, Jennifer A. Rode, and Kelly Waldman. 2011. Competing Online Viewpoints and Models of Chronic Illness.. In Proceedings of the SIGCHI Conference on Human Factors in Computing Systems (CHI '11). ACM Press, New York, NY, 589-598. https://doi.org/10.1145/1978942.1979027

[29] Mollie McKillop, Lena Mamykina, and Noémie Elhadad. 2018. Designing in the Dark: Eliciting Self-tracking Dimensions for Understanding Enigmatic Disease.. In Proceedings of the SIGCHI Conference on Human Factors in Computing Systems (CHI '18). ACM Press, New York, NY, 585:1-585:15. https://doi.org/10.1145/3173574.3174139

[30] Priya Nambisan. 2011. Information seeking and social support in online health communities: impact on patients' perceived empathy. fournal of the American Medical Informatics Association 18, 3 (May 2011), 298-304. https://doi.org/10.1136/amiajnl-2010-000058

[31] Lisa Neal, Kate Oakley, Gitte Lindgaard, David Kaufman, Jan Marco Leimeister, and Ted Selker. 2007. Online Health Communities.. In CHI '07 Extended Abstracts on Human Factors in Computing Systems (CHI EA '07). ACM Press, New York, NY, 2129-2132. https://doi.org/10. $1145 / 1240866.1240965$

[32] Elizabeth Sillence, Claire Hardy, Pam Briggs, and Peter R Harris. 2016. How do carers of people with multiple sclerosis engage with websites containing the personal experiences of other carers and patients? Health Informatics fournal 22, 4 (Dec 2016), 1045-1054. https://doi. org/10.1177/1460458215607938

[33] Meredith M. Skeels, Kenton T. Unruh, Christopher Powell, and Wanda Pratt. 2010. Catalyzing Social Support for Breast Cancer Patients.. In Proceedings of the SIGCHI Conference on Human Factors in Computing Systems (CHI '10). ACM Press, New York, NY, 173-182. https://doi. org $/ 10.1145 / 1753326.1753353$

[34] Karl E. Weick. 1995. Sensemaking in Organizations. SAGE, Thousand Oaks, CA.

[35] Jennifer L. Welbourne, Anita L. Blanchard, and Marla D. Boughton. 2009. Supportive Communication, Sense of Virtual Community and Health Outcomes in Online Infertility Groups.. In Proceedings of the Fourth International Conference on Communities and Technologies (C\&T '09). ACM Press, New York, NY, 31-40. https://doi.org/10.1145/ 1556460.1556466
[36] Xiaomu Zhou, Si Sun, and Jiang Yang. 2014. Sweet Home: Understanding Diabetes Management via a Chinese Online Community.. In Proceedings of the SIGCHI Conference on Human Factors in Computing Systems (CHI '14). ACM Press, New York, NY, 3997-4006. https://doi.org/10.1145/2556288.2557344

[37] Denniz Zolnoun, Eliza M. Park, Charity G. Moore, Cara A. Liebert, Frank F. Tu, and Sawson As-Sanie. 2008. Somatization and psychological distress among women with vulvar vestibulitis syndrome. International Journal of Gynecology \& Obstetrics 103, 1 (Oct 2008), 38-43. https://doi.org/10.1136/amiajnl-2010-000058 